\title{
Génesis de "Gracias a la vida": del canto íntimo desgarrado al canto universal
}

\author{
Benoît Santini \\ Université du Littoral Côte d'Opale \\ benoitsantini@orange.fr
}

\begin{abstract}
Resumen
Este artículo propone un estudio genético de una de las canciones más famosas de Violeta Parra, "Gracias a la vida", considerada a menudo testamento poético de la artista chilena. Poseído por el Museo Violeta Parra y expuesto en una de sus salas, el manuscrito de esta pieza, con sus tachados, reescrituras y remordimientos, da muestra de la búsqueda, por parte de la poeta, de la expresión más precisa del dolor amoroso, a la par que confirma el trabajo formal que efectúa a través del manejo de la metáfora.
\end{abstract}

\section{Palabras clave}

Violeta Parra, "Gracias a la vida", manuscrito, estudios genéticos, música.

\section{Gênese de "Gracias a la vida": do canto íntimo dilacerado no canto universal Resumo}

Este artigo propõe um estudo genético de uma das canções mais famosas de Violeta Parra, "Gracias a la vida", considerada amiúde testamento poético da artista chilena. Possuído pelo Museu Violeta Parra e exposto numa das suas salas, o manuscrito desta peça com os seus riscados, reescritas e remorsos, demonstra a procura, pela parte da poetisa, da expressão mais precisa da dor amorosa, ao mesmo tempo em que confirma o trabalho formal que realiza através do manejo da metáfora.

\section{Palavras-chave}

Violeta Parra, "Gracias a la vida", manuscrito, estudo genético, música.

\section{Genesis of "Gracias a la vida": from painfully intimate song to universal song}

\begin{abstract}
This article proposes a genetic criticism of "Gracias a la vida," one of Violeta Parra's most famous compositions and often considered her poetic testament. Preserved and exhibited at the Violeta Parra Museum, the manuscript of this song, with its deletions, rewritings and regrets, not only shows how Parra searched for a precise language to express love's sorrows, but also confirms her formal command of poetic metaphors.
\end{abstract}

\section{Keywords}

Violeta Parra, "Gracias a la vida", manuscript, genetic criticism, music. 
Benoît Santini. Génesis de "Gracias a la vida": del canto íntimo desgarrado al canto universal. 
Estudiar la génesis del poema-canción "Gracias a la vida", de Violeta Parra, lleva al investigador a interesarse por lo que Élida Lois llama "la interpretación del proceso escritural" (2008: 135). Efectivamente, dado que la génesis textual suele estudiar las metamorfosis del texto, cotejando versiones manuscritas, dactiloscritas e impresas, así como archivos pre-redaccionales, cuando se dispone de ellos, es apasionante poder analizar este mismo proceso por lo que se refiere a "Gracias a la vida", poema emblemático por ser una de las últimas creaciones musicales de la autora antes de su suicidio. Presente en el disco (LP) Las últimas composiciones, aparecido en 1966, esta canción compuesta en La Paz (Deuber, Ziegler y Divorne, 2016: 68) podría brindarnos datos relevantes acerca de cambios realizados a lo largo del proceso escritural que pudieran dar cuenta de remordimientos de la autora. No obstante, uno se enfrenta, en este caso, a una dificultad mayor: la ausencia de diferentes versiones manuscritas de "Gracias a la vida".

En efecto, según nuestras informaciones, sólo se dispone de una versión manuscrita única de la canción, exhibida en una vitrina de una sala del Museo Violeta Parra de Santiago de Chile, al lado de su guitarrón y con la indicación siguiente colocada a un costado por la fundación homónima: “'Gracias a la vida' Manuscrito Violeta Parra. 1964-1965 Facsímil". Este folio manuscrito ya está reproducido en El libro mayor de Violeta Parra, publicado en Madrid por Ediciones Michay, en la colección Libros del Meridión, el año 1985, acompañado de la indicación siguiente: "Facsímil parcial del texto manuscrito de 'Gracias a la vida'" (Parra, 1985: 144). El hecho de evocar el "texto manuscrito" de esta composición da la impresión de que sólo se conservó una versión de dicho texto. ¿Hubo otras versiones manuscritas y/o dactiloscritas? ¿Están guardadas en los cuadernos privados de la familia Parra? ¿Se destruyeron? No pudimos acceder a esta información e ignoramos, por tanto, si todavía existen otras versiones manuscritas y/o dactiloscritas mediante las cuales se podría recomponer el proceso creativo quizá completo de "Gracias a la vida" y de las composiciones de Violeta Parra, desde los primeros esbozos hasta la grabación del LP que contiene dicha canción. No obstante, este archivo único posee una serie de elementos que permiten proponer una primera tentativa de reconstrucción de la génesis de la composición.

¿Qué manuscritos de la cantautora pueden consultar los genetistas? Por ejemplo, en julio de 2017, el ingeniero Miroslav Skármeta obsequió quintetas manuscritas de Violeta al Museo Violeta Parra, enriqueciendo así el fondo de manuscritos autógrafos de la artista poseídos por la institución (Anónimo) ${ }^{1}$. Asimismo, en agosto de 2017, se realizó una muestra de arpilleras y manuscritos de canciones de la poeta en el centro cultural Gabriela Mistral (GAM) de Santiago de Chile (Saure, 2017) y la hija de la artista, Isabel Parra, posee, por su parte, un cuaderno manuscrito de las composiciones de su madre, como décimas autobiográficas y

1. Por ejemplo, se pueden consultar en una pantalla cuadernos de manuscritos digitalizados de la autora. Véase el sitio web del Museo Violeta Parra (2017). 
textos inéditos, recopilados en el libro Poesías, publicado por la académica Paula Miranda (Rivas, 2016, 25 de julio).

No se han realizado todavía análisis genéticos de la obra de Violeta Parra; el único estudio llevado a cabo a partir de un manuscrito de Violeta fue el "análisis grafológico del último manuscrito de Violeta Parra", redactado por el escritor y editor Óscar Villegas Brandau y publicado en la revista Araña gris en 1998. Se trata de un estudio del manuscrito de "La lavandera", considerada la última canción de Violeta, compuesta en diciembre de 1966. En este breve artículo de dos páginas y media, el autor se centra en el estado anímico de la poeta, reflejado en la letra, lo cual no parece muy útil a la hora de elaborar un estudio genético de la poesía de Violeta-aunque la depresión previa al suicidio del 7 de febrero de 1967 pueda aportar datos interesantes sobre el contenido del LP Las últimas composiciones. Recordemos que lo que le interesa al genetista es el proceso escritural, la evolución del texto, el estudio del antetexto, lo cual difiere del propósito de Villegas Brandau.

Por tanto, se pretende en este artículo elaborar un primer acercamiento a la génesis de "Gracias a la vida". ¿Qué informaciones brinda el manuscrito sobre el proceso de escritura de esta composición y qué elementos resultan particularmente pertinentes para elaborar una reflexión acerca de éste? Para contestar esta pregunta, las pistas de análisis del presente texto abordarán tanto el vínculo entre la biografía de la poeta y su creación artística como la importancia de la prosodia y del ritmo que llevan a la poeta a efectuar minuciosamente una serie de cambios en la letra de su canción.

\section{Historia de la canción}

Acerca del título de "Gracias a la vida", Paula Miranda recuerda que, "según testimonia Liliana Soleney, habría sido su madre, la señora Carmen Rojas Pozo, gran amiga de Violeta en Santiago, quien le habría transmitido a la artista esta frase: 'mi madre usaba frecuentemente esta expresión... [Violeta] cogió sus creaciones de las vivencias de muchos y las verbalizó en su guitarra' [...]" (Miranda, 2013: 142). Violeta se nutre entonces de sus relaciones amistosas, familiares, amorosas, las que constituyen una rica fuente de inspiración para sus composiciones. Asimismo, nos enteramos de que "biográficamente esta canción fue compuesta por Violeta poco tiempo después de su primer intento de suicidio" (Miranda, 2013: 143): eso significa que, si consideramos que, para Miranda, este primer intento ocurrió en enero de 1966, como lo escribe Niño Vásquez y lo confirma el artículo de La Tercera "Violeta Parra, de dicha y quebranto" (2009, 3 de octubre), la composición de la canción se podría fechar después del 14 enero de 1966 (Niño Vásquez, 2015: 24). Sin embargo, Erica Deuber Ziegler y Ana Divorne (2016: 67) aluden a una tentativa de suicidio anterior con barbitúricos que habría ocurrido en febrero de 1965 en 
Santiago. Fechar precisamente la composición de la canción es entonces un reto, dadas las informaciones discordantes acerca de la primera tentativa de suicidio de Violeta. ¿No habría podido componer entonces esta pieza entre febrero de 1965 y enero de 1966? Recordemos, como escribe Nelson Niño Vásquez, que

En octubre de 1960 Violeta Parra comenzó una relación sentimental con el antropólogo suizo Gilbert Favré (sic), quien era dieciocho años menor que ella. En 1961 ambos viajaron a París y decidieron permanecer allí por cuatro años, período durante el cual Favré aprendió a interpretar varios instrumentos musicales con ayuda de Violeta, hasta que se convirtió en un virtuoso ejecutante de quena. Luego de su regreso a Santiago de Chile en 1965 la relación entre ambos llegó a su fin. El antropólogo suizo partió rumbo a Bolivia y Violeta cayó en una profunda crisis emocional (Niño Vásquez, 2015: 23-24).

Así, si el título de "Gracias a la vida" le fue inspirado por Carmen Rojas Pozo, el contenido, considerado a menudo irónico por agradecer a la vida antes de suicidarse, se relacionaría con la "crisis emocional" vivida por Violeta, en especial tras su ruptura con Favre:

Las canciones compuestas por Violeta Parra durante el año 1966 ciertamente reflejan su convulsionado estado emocional, canciones que forman parte del histórico álbum publicado por ella hacia fines de ese mismo año. [...] Comenzó a ser distribuido comercialmente a mediados del mes de enero de 1967 y la propia Violeta tuvo oportunidad de vender varios ejemplares durante sus últimas presentaciones (Niño Vásquez, 2015: 24).

1966 es entonces un año clave en la producción artística y las vivencias personales de Violeta. Viaja a La Paz, con el objetivo de regresar a Chile con Favre. En ese entonces, éste toca la quena con Los Jairas en la Peña Naira donde vive una relación sentimental con una muchacha boliviana, situación de la que se entera Violeta (Niño Vásquez, 2015: 24). Se tendrían entonces mayores precisiones acerca de la fecha de composición de "Gracias a la vida" siguiendo las aclaraciones citadas más arriba de Deuber y Divorne. Por consiguiente, además de la influencia y de la inspiración amistosa y amorosa para la elaboración de "Gracias a la vida", sería preciso relevar la importancia del desplazamiento y la estadía en Bolivia desde donde la artista compone su famosísima canción.

Antes de adentrarse en el manuscrito de la canción, es interesante hacer referencia a lo que explica en una entrevista Luis Torrejón, quien, en su estudio, grabó el último LP de Violeta:

Las últimas composiciones, sin embargo, se grabaron en sesiones que "generalmente eran de día, tipo tres o cuatro de la tarde", en los amplios estudios que RCA Víctor tenía en el sexto piso de un edificio ubicado en Matías Cousiño 150, en pleno centro de Santiago. Fue en los últimos meses de 1966, cuando Violeta Parra ya había vuelto de Europa y había instalado su carpa en La Reina. [...] En Las últimas composiciones, Violeta Parra es acompañada en varias canciones por sus hijos, Ángel e Isabel, y por 
el uruguayo Alberto Zapicán. En la edición original, éstos aparecen a cargo del acompañamiento en "guitarra, guitarrilla, charango y bombo". El segundo instrumento, en realidad, es un cuatro (Alarcón, 2017, 3 de octubre).

Por lo que se refiere precisamente a la canción que nos ocupa, Torrejón precisa que, "en el LP, "Gracias a la vida", por ejemplo, acá está primero, pero se grabó en la cuarta sesión, yo creo. Hicimos cinco sesiones de tres horas cada una, más o menos". Estas precisiones son importantes, en la medida en que iluminan el contexto de creación y de grabación de una de las composiciones favoritas de Violeta Parra, según explica en Punta Arenas en 1966:

Creo que las canciones más lindas, las más maduras (perdónenme que les diga canciones lindas, habiéndolas hecho yo, pero qué quieren ustedes, soy huasa y digo las cosas sencillamente como las siento), las canciones más enteras que he compuesto son: "Gracias a la vida", "Volver a los diecisiete" y "Run run se fue pa'l norte".

Yo estoy contenta de considerarme en este momento como compositora (Subercaseaux, Stambuk, Londoño, 1984: 32-33).

\section{El manuscrito}

El folio disponible en la vitrina de la fundación Violeta Parra es de papel blanco y de tamaño A4 $(21 \times 29,7 \mathrm{~cm})$. El texto manuscrito se compone de seis quintetos y un verso final ${ }^{2}$. Las estrofas 1-2 y 2-3 vienen separadas por una línea corta. El texto está escrito con tinta negra y el papel posee algunas manchas de humedad $u$ hongos. Asimismo, se descubren dos tachados que cubren enteramente dos de los versos y tachados menores de sustitución. En cambio, no viene acompañada la letra de la canción de notas musicales o de una partitura, ni de la indicación de los acordes utilizados por Violeta Parra. La poeta se centra, en este caso, en el texto en sí.

La versión que se puede consultar es una versión ya avanzada del proceso escritural de la canción. Forma parte de la "fase redaccional" y de la etapa de "textualización", según la terminología de Pierre-Marc de Biasi (2011: 89). Gracias a la limpieza de la mayor parte de las estrofas y la ausencia de numerosos tachados, así como la estructura en seis estrofas igual que en la versión grabada, suponemos que no se trata de la primera versión del texto. Sería útil, entonces, poder cotejar las distintas versiones manuscritas de "Gracias a la vida", si es que existen o existieron, e incluso de los manuscritos de Las últimas composiciones para entender mejor la coherencia del proceso escritural de la poeta: ¿en qué soportes compuso estas canciones, con qué tinta, en qué lapso de tiempo? ¿Hubo desplazamientos de versos de un poema a otro?

2. Juan Pablo González escribe que Violeta se inspira de la sirilla, género musical de la isla de Chiloé, compuesta de seis octavos, para crear su canción construida con seis quintetos (González, 2017: s.p.). 
Para volver al manuscrito de la canción, la ausencia de fecha salta a la vista también. Como ya se dijo más arriba, el poema habría sido escrito en torno a abrilmayo de 1966 pero ¿a cuándo se remonta el proyecto de canción? ¿Se procedió a una "investigación preliminar" (De Biasi, 2011: 80) con ideas previas, fragmentos de redacción o planes exploratorios escritos en cuadernos? ¿Hubo lecturas preparatorias, intentos redaccionales, guión global en la "inicialización del proyecto" (81), sea de la canción en sí, sea del conjunto de las canciones del LP? ${ }^{3}$ La consulta de la correspondencia de Violeta, en particular de cartas enviadas a Favre en ese entonces, puede iluminar sobre la génesis de "Gracias a la vida". En efecto, en El libro mayor de Violeta se transcribe una carta sin fecha de Violeta a Favre, pero cuyo contenido permite adivinar que fue escrita en torno a 1963 -alude a los conciertos en París con sus hijos y a los tres años que llevan juntos Gilbert y ella-; en ésta, se lee:

Es terrible la vida. Yo quisiera estar allí, pero estoy aquí. Yo siento que quiero un hombre, pero mi trabajo me aplana. Dolorosamente tomo un tren que me aleja de ti, pero lo tomo sin melodrama, sin debilidad, sin dudarlo ningún momento, con la cabeza llena de ti, con el cuerpo lleno de tu huella (Parra, 1985: 96).

Estas frases, en particular la primera, "Es terrible la vida", constituiría la cara oscura de la imagen que tiene Violeta de la existencia, mientras que "Gracias a la vida", escrita tres años después, sería el reverso "luminoso" de esta misma imagen. Se pasaría entonces de "Es terrible la vida" a "Gracias a la vida", o sea, de una concepción dolorosa de la existencia a una conciencia de la belleza del vivir. La vida, que constituye un motivo temático clave en diversas composiciones de la poeta, encontraría en "Gracias a la vida" su esencia e incluso su quintaesencia: se puede pensar en las canciones "A la una", "Arranca, arranca pericona", "La cueca de los poetas", "Cuecas del libro", "El amor", "El joven de casarse", "Epílogo", "Hijo que tiene a sus padres", "La denuncia", "La muerte", "La renuncia", "LXXXI", "Paloma ingrata", "Run Run se fue pa'I norte", etc. Paradójicamente, esta obsesión por la vida desemboca en un suicidio, lo que explica que algunos investigadores consideren a la canción como testamento artístico de la poeta.

Antes de analizar las metamorfosis del texto (tachados, tiempos verbales, entre otros), es oportuno realizar una puesta en relación de la versión manuscrita y de la versión grabada ${ }^{4}$ :

3. Estas operaciones conciernen más bien al estudio de manuscritos novelísticos. No obstante, en el caso de la poesía, no resulta aberrante manejar esta terminología. Refirámonos por ejemplo al caso del poeta Raúl Zurita quien, al preparar la redacción de su libro La Vida Nueva (1994), realiza investigaciones preliminares, escribe guiones y dibuja croquis en cuadernos, como se puede observar en los abundantes manuscritos de la colección Carlos Alberto Cruz. Aunque el caso de Violeta Parra sea distinto, nos parece pertinente plantearnos por lo menos estas preguntas.

4. Ponemos en negrita fragmentos que evolucionaron entre el manuscrito y la versión grabada. 


\begin{tabular}{|c|c|}
\hline Versión manuscrita & Versión grabada \\
\hline $\begin{array}{l}\text { Gracias a la vida que me ha dado tanto } \\
\text { me ha dado los ojos con que estoy mirando } \\
\text { con ellos distingo lo negro del blanco } \\
\text { y en el alto cielo su fondo estrellado } \\
\text { y en las multitudes el hombre que yo amo. } \\
*\end{array}$ & $\begin{array}{l}\text { Gracias a la vida que me ha dado tanto } \\
\text { Me dio dos luceros que cuando los abro } \\
\text { Perfecto distingo lo negro del blanco } \\
\text { Y en el alto cielo su fondo estrellado } \\
\text { Y en las multitudes el hombre que yo amo. }\end{array}$ \\
\hline $\begin{array}{l}\text { Gracias a la vida que me ha dado tanto } \\
\text { me ha dado el oído que llevo escuchando } \\
\text { no pierdo detalle grillos y canarios } \\
\text { martillos turbinas ladridos chubazcos [sic] } \\
\text { y la voz tan tierna de mi bien amado }\end{array}$ & $\begin{array}{l}\text { Gracias a la vida que me ha dado tanto } \\
\text { Me ha dado el oído que en todo su ancho } \\
\text { Graba noche y día, grillos y canarios } \\
\text { Martillos, turbinas, ladridos, chubascos } \\
\text { Y la voz tan tierna de mi bien amado }\end{array}$ \\
\hline $\begin{array}{l}\text { Gracias a la vida que me ha dado tanto } \\
\text { me ha dado el sonido con que estoy } \\
\text { [eantando hablando } \\
\text { con él las palabras que voy deletreando } \\
\text { madre amigo hermano y luz alumbrando, } \\
\text { ta ruta del alma del que estoy amando } \\
\text { ta duta delatma del que estoy amando }\end{array}$ & $\begin{array}{l}\text { Gracias a la vida que me ha dado tanto } \\
\text { Me ha dado el sonido y el abecedario } \\
\text { Con él las palabras que pienso y declaro } \\
\text { Madre amigo hermano y luz alumbrando, } \\
\text { La ruta del alma del que estoy amando. }\end{array}$ \\
\hline $\begin{array}{l}\text { Gracias a la vida que me ha dado tanto } \\
\text { me ha dado la marcha de mis pies cansados } \\
\text { con ellos anduve ciudades y charcos, } \\
\text { desiertos y playas montañas y llanos } \\
\text { ta ruta del alma del que estoy amando } \\
\text { Gracias a la vida que me ha dado tanto } \\
\text { me ha dado dio el corazón que está palpitando } \\
\text { cuando miro el fruto del cerebro humano } \\
\text { cuando miro el bueno tan lejos del malo } \\
\text { cuando miro el fondo de tus ojos claros }\end{array}$ & $\begin{array}{l}\text { Gracias a la vida que me ha dado tanto } \\
\text { Me ha dado la marcha de mis pies cansados } \\
\text { Con ellos anduve ciudades y charcos, } \\
\text { Playas y desiertos montañas y llanos } \\
\text { Y la casa tuya, tu calle y tu patio. } \\
\text { Gracias a la vida que me ha dado tanto } \\
\text { Me dio el corazón que agita su marco } \\
\text { Cuando miro el fruto del cerebro humano, } \\
\text { Cuando miro al bueno tan lejos del malo, } \\
\text { Cuando miro al fondo de tus ojos claros. }\end{array}$ \\
\hline $\begin{array}{l}\text { Gracias a la vida que me ha dado tanto } \\
\text { me ha dado la risa y me ha dado el llanto } \\
\text { con esto distingo dicha de quebranto } \\
\text { los dos materiales que forman mi canto } \\
\text { y el canto de Udes. que es el mismo canto }\end{array}$ & $\begin{array}{l}\text { Gracias a la vida que me ha dado tanto } \\
\text { Me ha dado la risa y me ha dado el llanto, } \\
\text { Así yo distingo dicha de quebranto } \\
\text { Los dos materiales que forman mi canto } \\
\text { Y el canto de ustedes que es el mismo canto } \\
\text { Y el canto de todos que es mi propio canto. }\end{array}$ \\
\hline Gracias a la vida que me ha dado tanto & $\begin{array}{l}\text { Gracias a la vida } \\
\text { Gracias a la vida } \\
\text { Gracias a la vida } \\
\text { Gracias a la vida }\end{array}$ \\
\hline
\end{tabular}




\section{¿Qué nos dicen las metamorfosis del texto?}

Almuth Grésillon dice acerca de los tachados de autor: "la tachadura es una borradura visible, un silencio audible y, por tanto, una huella legible"5 (1996: s.p.). Son pocos los tachados del manuscrito de "Gracias a la vida", pero, como escribe la misma Grésillon acerca de un poema de Supervielle, "todavía subsisten puntos de inestabilidad que son retrabajados"6 (1998: 87). En particular, llama la atención el verso "La ruta del alma del que estoy amando", tachado tres veces y colocado en las estrofas 3 y 4 del manuscrito. ¿Qué significa el tachado? ¿Una insatisfacción de la artista? ¿Por qué, finalmente, decidió conservarlo? Sin reducir la letra de la canción a lo estrictamente autobiográfico ${ }^{7}$, se podría considerar que la ruptura con Favre, ocurrida poco tiempo antes, en enero de 1966, se integra al mismo manuscrito mediante el tachado que niega el afecto de la hablante-aquí, encarnación textual de la poeta- por el hombre al que está amando. Tachar, en este caso, reproduce la herida de amor, o sea, lo que Violeta vivió como un verdadero aniquilamiento: la ruptura amorosa. Estos versos añadidos adquieren el estatuto de tachados de sustitución, antes de verse a su vez eliminados-aunque, en el primer caso, se trate de sustituir el verso por otro idéntico y, en el segundo caso, de sustituir "y la casa tuya, tu calle y tu patio" por el verso "La ruta del alma del que estoy amando", convirtiéndose el espacio geográfico y la vía de comunicación ("casa", "calle", "patio") en espacio mental ("ruta del alma"). La segunda persona del singular viene reemplazada por una primera persona en el verso reescrito, con lo cual el Yo lírico opta por centrarse en su propia situación. Así, el verso se desplaza, yerra, no encuentra su lugar y refleja las dudas de la voz poética, errabunda, perdida, sin punto fijo, aunque finalmente la poeta decide adoptarlo y conservarlo en la versión definitiva.

Si el tachado tiene como meta la de sustituir un verso previo, se procede también al añadido de un verso final en la versión grabada, verso ausente en la versión manuscrita, "Y el canto de todos que es mi propio canto", siendo la última estrofa un sexteto (canción grabada). Esta apertura a la humanidad entera no figura en el manuscrito; el canto desgarrado era, quizá, mucho más íntimo en los primeros esbozos y se vuelve universal al cabo del proceso escritural. Violeta Parra también efectúa unas sustituciones de versos o palabras, manifestación fehaciente de los tanteos de la escritora. Entre ellos, descubrimos sustituciones relevantes, en las cuales queremos hacer hincapié, y otras más anecdóticas que no analizaremos. Así, el verso "me ha dado los ojos con que estoy mirando" se convierte en "Me dio dos luceros que cuando los abro"; se reemplaza entonces la mención a "los

\footnotetext{
5. "Ia rature est un effacement visible, un silence audible, et donc, une trace lisible", en francés.

6. "subsistent encore des points d'instabilité qui sont retravaillés", en francés.

7. Recordemos, sin embargo, la importancia del elemento autobiográfico en las composiciones de la artista; citemos, por ejemplo, el libro Décimas: autobiografía en versos, publicado en 1976 por la editorial Pomaire con introducción de Pablo Neruda, Pablo de Rokha y Nicanor Parra.
} 
ojos" por la metáfora "dos luceros", como si el cuerpo de la hablante se esfumara, se convirtiera en mera metáfora, perdiera de su consistencia y así se produjera un suicidio progresivo de la voz lírica mediante su fragmentación. También se nota que el cambio se debe al deseo de conservar la estructura de la subordinada relativa recurrente introducida por el pronombre "que". Así, para evitar una ruptura en la coherencia global, Violeta hace desaparecer la estructura "con que", conservando la acentuación mayoritaria en la segunda y quinta sílabas del hemistiquio, y suprime el pretérito perfecto, optando por el uso del pretérito indefinido ${ }^{8}$. Otro ejemplo es muy evocador: si en el manuscrito se lee "Me ha dado/dio el corazón que está palpitando", en la grabación se escucha "me dio el corazón que agita su marco". La desaparición del verbo "palpitar", sustituida por la perífrasis "agitar su marco", posee una resonancia peculiar, tanto más cuanto que sabemos que, unos meses más tarde, Violeta se dará la muerte. Pero también la evocación concreta se convierte en metáfora y refleja, pues, la minuciosidad de la autora y su profundo trabajo formal. Se pueden citar asimismo las metamorfosis del verso "me ha dado el sonido con que estoy eantando hablando", que se transforma en la versión definitiva en "Me ha dado el sonido y el abecedario". Se procede a una primera sustitución en el mismo manuscrito mediante un tachado y una reescritura, dado que de "cantando" se pasa a "hablando"; el canto se presenta entonces como una historia que se cuenta $-\mathrm{y}$ se interpreta-, recurso frecuente en las composiciones de este último LP de Violeta9 .

En cuanto a las sonoridades, la aliteración inicial de la oclusiva [k] en el segundo hemistiquio ("con", "que", "cantando"), muy abrupta, se suaviza con la sustitución de "cantando" por "hablando". Del manuscrito a la versión definitiva se constata que la palabra viene sustituida por la herramienta que permite crear la palabra y el canto: el abecedario. El fenómeno inverso se produce con el paso de "Las palabras que voy deletreando" a "las palabras que pienso y declaro": la hablante evolucionó entre la versión manuscrita y la definitiva, pasando de la actividad de "deletreo" que puede hacer un niño al comienzo de su aprendizaje de la lectura a una madurez de reflexión y de creación, expresada a través de los verbos "pensar y declarar", los que, además, hacen hincapié en el paso de la etapa de la elaboración mental a la etapa de expresión oral. Se crea un hemistiquio con una división perfecta ( $3+3$ sílabas), estableciendo así un equilibrio fónico. La cantautora da muestra, otra vez, de su precisión y su rigor, en cuanto al trabajo verbal de su composición. Estos ejemplos revelan, por último, la voluntad de aligerar el verso, suprimiendo los gerundios y privilegiando el empleo de presentes de indicativo que preservan la actualización el enunciado.

8. Efectivamente, en el hemistiquio "Me ha dado los ojos", la acentuación se encuentra en la segunda sílaba, dada la presencia de una sinalefa, y en la quinta.

9. Por ejemplo, en "Run Run se fue pa'I norte", la hablante explica: "me dice que su viaje / se alarga más y más [...] / y cuenta una aventura / que paso a deletrear", y "El Albertío" se escucha: "Alberto, sijo, me llamo". 


\section{"Notas" finales}

Este estudio genético que tiene en cuenta una de las cartas de Violeta y diversos poemas suyos anteriores a "Gracias a la vida" cuyo motivo recurrente es la vida -aunque consideramos que podría enriquecerse dicho análisis con la consulta de otros manuscritos elaborados en el proceso de creación de esta canción- demuestra la paradoja de un canto a la vida en el que la evocación concreta del cuerpo da paso a la metáfora, unida a la elaboración de un trabajo formal preciso. Entre el manuscrito y la versión grabada, el texto se metamorfosea, se tacha en parte; en él se borran diversas alusiones al cuerpo en un proceso de autodestrucción acentuado por el ritmo lancinante y el uso del charango. Fernando Sáez, relacionando la creación de "Gracias a la vida" con la muerte inminente de Violeta, escribe que, "compuesta luego del intento de suicidio de enero del '66, [...] lejos de ser un himno a la vida, es un recuento poético de sus pérdidas" (Sáez, 1999: 159-160). No obstante, las metamorfosis del texto también pueden simplemente deberse, en algunos casos, a un trabajo sobre la musicalidad y el ritmo: así, se invierte el orden de las palabras del verso manuscrito "Desiertos y playas", que se convierte en "playas y desiertos" en la canción grabada. Por lo tanto, a la estructura "Gracias a la vida" ( 2 sílabas $+1+1+2)$ responde la estructura "Playas y desiertos" $(2+1+3)$.

Esta canción se caracteriza por las múltiples versiones señaladas por Niño Vásquez: "Ésta es también su canción más reconocida, la cual cuenta con cientos de versiones editadas en decenas de países, entre las que se destacan las interpretaciones de Mercedes Sosa, Plácido Domingo, Milva, Joan Báez, Omara Portuondo, Isabel Parra y la propia Violeta" (2015: 25). En algunos casos, se retoca ligeramente la letra, como en la versión de "Voces unidas por Chile" (2010), grupo compuesto por Juanes, Alejandro Sanz, Juan Luis Guerra, Laura Pausini, Fher (Maná), Shakira, Michael Bublé, Miguel Bosé y Beto Cuevas y con un objetivo caritativo: ayudar a los damnificados del terremoto chileno de 2010. En esta versión, "el hombre que yo amo" viene sustituido por "la mujer que amo" y se añaden los versos "Gracias a la vida que nos da risas y llantos / Gracias a la vida que nos une en este canto". De escritura en reescritura, de interpretaciones en reinterpretaciones, esta canción se presenta como una obra en evolución permanente y que continúa su vida a pesar del destino trágico de su intérprete.

\section{Referencias}

Alarcón, R. (2017, 3 de octubre). Luis Torrejón, el hombre que grabó Las últimas composiciones de Violeta Parra. DiarioUchile (sitio web). Recuperado el 09 de abril de 2018 de http://radio.uchile.cl/2017/10/03/luis-torrejon-elhombre-que-grabo-las-ultimas-composiciones-de-violeta-parra/.

Archivos Chile (2003-2007.). Violeta Parra (1917-1967). Archivos Chile (sitio web). Recuperado el 25 de noviembre de 2018 de http://www.archivochile.com/ Cultura_Arte_Educacion/vp/d/vpde0005.pdf 
De Biasi, P.-M. (2011) Génétique des textes. París: CNRS.

Deuber Ziegler, E., Divorne, A. (2016). Alfredo Domínguez en el V centro de grabado contemporáneo de Ginebra (CGCG). Fuentes. Revista de la Biblioteca y Archivo Histórico de la Asamblea Legislativa Plurinacional, 10(47). La Paz: Biblioteca y Archivo Histórico de la Asamblea Legislativa Plurinacional, 6677.

González, J. P. (2017). Lo popular, la música popular y sus tensiones. En: Colectivo. Violeta Parra. Después de vivir un siglo. Santiago de Chile: CNCA.

Grésillon, A. (1998). La critique génétique à l'œuvre. Étude d’un dossier génétique: "Vivre encore" de Jules Supervielle. En: Contat, M.; Ferrer, D.; dir. Pourquoi la critique génétique? París: CNRS, 61-93.

Grésillon, A. (1996). Raturer, rater, rayer, éradiquer, radier, irradier. Institut des textes et manuscrits modernes. Recuperado el 09 de abril de 2018 de http:// www.item.ens.fr/index.php?id=13973.

La Tercera (2009, 3 de octubre). Violeta Parra, de dicha y quebranto. La Tercera [sitio web]. Recuperado el 05 de noviembre de 2018 de http://www2.latercera.com/noticia/violeta-parra-de-dicha-y-quebranto/.

Lois, É. (2008). Las técnicas filológicas y las innovaciones técnicas de la genética textual. En: Colla, F., ed. Archivos. Cómo editar la literatura latinoamericana del siglo XX. Poitiers: CRLA-Archivos.

Miranda, P. (2013). La poesía de Violeta Parra. Santiago de Chile: Cuarto Propio.

Museo Violeta Parra (2017). Museo Violeta Parra agradeció a las instituciones y personas que espontáneamente le han legado obras relativas a la artista chilena. Museo Violeta Parra (sitio web). Recuperado el 09 de abril de 2018 de http://museovioletaparra.cl/este-martes-museo-violeta-parra-agradece-a-las-instituciones-y-personas-que-espontaneamente-le-han-legadoobras-relativas-a-la-artista-chilena/.

Niño Vásquez, N. (2015). Gracias a la vida, Trío para un nuevo tiempo y el tercer período compositivo de Celso Garrido-Lecca. Revista musical chilena, 69(223), 21-46. https://dx.doi.org/10.4067/S0716-27902015000100003.

Parra, I. (1985). El libro mayor de Violeta Parra. Madrid: Michay.

Rivas, M. (2016, 25 de julio). El inagotable caudal poético de Violeta Parra que Chile se demoró en reconocer. El Mostrador (sitio web). Recuperado el 09 de abril de 2018 de http://www.elmostrador.cl/cultura/2016/07/25/ el-inagotable-caudal-poetico-de-violeta-parra-que-chile-se-demoro-enreconocer/.

Sáez, F. (1999). La vida intranquila: Violeta Parra, biografía esencial. Santiago de Chile: Sudamericana.

Saure, G. (2017). Exposición revela arpilleras y manuscritos inéditos de Violeta Parra. La Nación (sitio web). Recuperado el 08 de abril de 2018 de http:// 
Panambí n. 7 Valparaíso dic. 2018 ISSN 0719-630X. 59-71. DOI:10.22370/panambi.2018.7.1133.

lanacion.cl/2017/08/21/exposicion-revela-aspilleras-y-manuscritos-ineditos-de-violeta-parra/.

Subercaseaux, B.; Stambuk, P.; Londoño, J. (1984). La bicicleta. Revista cultural. Gracias a la vida. Violeta Parra testimonio. 39 parte La carpa de la reina. Santiago: Granizo. 